\title{
Pacific
}

Journal of

Mathematics

\section{HELICOIDAL FLAT SURFACES IN HYPERBOLIC 3-SPACE}

Antonio Martínez, JoÃo Paulo dos Santos And Keti Tenenblat 


\title{
HELICOIDAL FLAT SURFACES IN HYPERBOLIC 3-SPACE
}

\author{
Antonio Martínez, João Paulo dos Santos and Keti Tenenblat
}

\begin{abstract}
A flat surface in hyperbolic space $\mathbb{T}^{3}$ is determined by a harmonic function as well as by its meromorphic data. In this paper, helicoidal flat surfaces in $\mathbb{H}^{3}$ are considered. A complete classification of the helicoidal flat fronts is given in terms of their hyperbolic Gauss maps as well as by means of linear harmonic functions. A family of examples that provides the classification of the helicoidal flat fronts is included. Moreover, it is shown that a flat surface in $\llbracket^{3}$ that corresponds to a linear harmonic function is locally congruent to a helicoidal flat front or to a peach front.
\end{abstract}

\section{Introduction}

The study of flat surfaces in hyperbolic 3-space has received much attention in the last few years, mainly because Gálvez, Martínez and Milán [Gálvez et al. 2000] have shown that flat surfaces in hyperbolic 3-space admit a Weierstrass representation formula in terms of meromorphic data as in the theory of minimal surfaces in $\mathbb{R}^{3}$.

It is known that the only complete examples are the horospheres and the hyperbolic cylinders (see [Spivak 1979]). Thus, a study of flat surfaces with singularities became essential for the advancement of the theory. An important contribution was given in [Kokubu et al. 2005; 2004], where an extension of the Weierstrass representation for flat surfaces with admissible singularities was introduced. Such surfaces are called flat fronts.

Helicoidal surfaces arise as a generalization of rotational surfaces and conical surfaces. They are invariant by a subgroup of the group of space isometries, called the helicoidal group, defined by a translation composed with rotation around an axis in the same direction. Rotational flat surfaces were classified in [Kokubu et al. 2004] in terms of meromorphic data.

The main purpose of this paper is to give a complete classification of the helicoidal flat surfaces in $\mathbb{H}^{3}$ in terms of meromorphic data as well as by means of linear

Research partially supported by the Ministério de Educação, CAPES/DGU Proc. No. 246/11, Brazil; Ministerio de Ciencia e Innovación MTM2010-1982, Spain; and Junta de Andalucía Grant FQM325. MSC2010: 53A35, 53C42.

Keywords: helicoidal surfaces, flat surfaces, flat fronts, hyperbolic space, conformal representation. 
harmonic functions. We construct a family of examples, which we call classifying examples, that provide the complete classification of the helicoidal flat surfaces. These results extend those obtained previously in [Kokubu et al. 2004] for rotational flat surfaces. Moreover, we characterize the flat fronts in $\mathbb{H}^{3}$ that correspond to linear harmonic functions.

The paper is organized as follows:

In Section 2, we give a brief description of helicoidal flat surfaces in $\mathbb{H}^{3}$ and present two particular cases of this class of surfaces, namely, the rotational and the conical flat surfaces.

In Section 3, we recall the well known result that in a neighborhood of a nonumbilic point, any flat surface in $\mathbb{\boxplus}^{3}$ admits a local parametrization that diagonalizes both the first and second fundamental forms determined by a (euclidean) harmonic function. We then present the conformal representation for flat fronts described in [Corro et al. 2010; Kokubu et al. 2005] and use it to characterize when a complex parameter diagonalizes both the first and second fundamental forms in terms of the hyperbolic Gauss maps. Moreover, we relate the harmonic function to the hyperbolic Gauss maps.

In Section 4, we describe a family of flat fronts that we call classifying examples whose hyperbolic Gauss maps are determined by a nonzero complex number, and we obtain the corresponding harmonic function.

Finally, in Section 5, we prove that a flat front in $\mathbb{H}^{3}$ is helicoidal if and only if it is locally congruent to one of the classifying examples. Moreover, we obtain a complete classification of the helicoidal flat fronts in terms of their hyperbolic Gauss maps as well as by means of suitable linear harmonic functions. We conclude by showing that any flat surface in $\mathbb{H}^{3}$ that corresponds to a linear harmonic function is locally congruent either to a helicoidal flat surface or to a so-called peach front.

\section{Helicoidal surfaces}

Helicoidal surfaces arise as a natural generalization of rotational surfaces. They are invariant under a one-parameter group of isometries obtained by composing a translation in a given direction with a rotation about an axis in the same direction. We consider the half-space model of the hyperbolic 3-space, that is,

$$
\mathbb{H}^{3}=\left\{\left(y_{1}, y_{2}, y_{3}\right) \in \mathbb{R}^{3}: y_{3}>0\right\}
$$

endowed with the metric

$$
\langle,\rangle=\frac{1}{y_{3}^{2}}\left(d y_{1}^{2}+d y_{2}^{2}+d y_{3}^{2}\right),
$$

with ideal boundary $\mathbb{C}_{\infty}=\left\{\left(y_{1}, y_{2}, 0\right) \mid y_{1}, y_{2} \in \mathbb{R}\right\} \cup\{\infty\}$. 
The helicoidal group relative to the $y_{3}$-axis is given as the composition

$$
h_{t}=\left(\begin{array}{ccc}
e^{\beta t} & 0 & 0 \\
0 & e^{\beta t} & 0 \\
0 & 0 & e^{\beta t}
\end{array}\right)\left(\begin{array}{ccc}
\cos \alpha t & -\sin \alpha t & 0 \\
\sin \alpha t & \cos \alpha t & 0 \\
0 & 0 & 1
\end{array}\right)
$$

of a rotation around the $y_{3}$-axis with angular pitch $\alpha$ with a hyperbolic translation of ratio $\beta$.

Every helicoidal surface can be generated by a suitable curve $\gamma: I \rightarrow \mathbb{H}^{3}$ by taking the composition

$$
\psi(t, s)=\left(h_{t} \circ \gamma\right)(s) .
$$

Notice that the curve $\gamma$ is chosen suitably so that (2-1) is a regular surface.

In order to have the helicoidal surface (2-1) flat, one has to require conditions on the curve $\gamma$, as in the following particular cases:

(i) Rotational flat surfaces $(\beta=0)$. We start with a curve parametrized by arc length on the plane $\left\{y_{2} \equiv 0\right\}$. It follows from the Gauss equation that the remaining coordinates, $y_{1}$ and $y_{3}$, must satisfy the differential equation

$$
\left(\frac{y_{1}}{y_{3}}\right)^{\prime \prime} \frac{y_{3}}{y_{1}}=0
$$

[do Carmo and Dajczer 1983; Spivak 1979], giving us the relation $y_{1}(s)=$ $(a s+b) y_{3}(s)$.

(ii) Conical flat surfaces $(\alpha=0)$. In this case, we just have a movement of translation. We can start with a curve on the horosphere $\left\{y_{3}=c\right\}$, where $c>0$ is a constant. Assuming that $c=1$, we consider the curve

$$
\gamma(s)=(r(s) \cos \theta(s), r(s) \sin \theta(s), 1),
$$

parametrized by the arc length, that is,

$$
\left(r^{\prime}\right)^{2}+\left(r \theta^{\prime}\right)^{2}=1
$$

Then the surface is flat if and only if one has the following expression for $r$ :

$$
r(s)=\sqrt{(a s+b)^{2}-1} .
$$

Therefore, we see that helicoidal surfaces arise as a generalization of the rotational and the conical surfaces, which are well known flat examples. We want to describe all the helicoidal flat surfaces in hyperbolic 3-space. 


\section{Conformal representation}

In this section, we characterize the flat surfaces in $\mathbb{M}^{3}$ by means of their first and second fundamental forms. We start by recalling that on a neighborhood of a nonumbilic point, any flat surface in $\mathbb{H}^{3}$ admits a local parametrization that diagonalizes both the first and second fundamental forms, which are determined by a (euclidean) harmonic function. We then consider the conformal representation for flat fronts and characterize a complex parameter that diagonalizes both the first and second fundamental forms in terms of the hyperbolic Gauss maps. We also relate the harmonic function to these maps.

It is well known that on a neighborhood of a nonumbilical point, a flat surface in $\mathbb{M}^{3}$ can be parametrized by lines of curvature, so that the first and second fundamental forms are given by

$$
\begin{aligned}
\mathrm{I} & =\cosh ^{2} \phi(u, v)(d u)^{2}+\sinh ^{2} \phi(u, v)(d v)^{2}, \\
\mathrm{II} & =\sinh \phi(u, v) \cosh \phi(u, v)\left((d u)^{2}+(d v)^{2}\right),
\end{aligned}
$$

where $\phi$ is a harmonic function, that is, $\phi_{u u}+\phi_{v v}=0$ (for details, see [Tenenblat 1998, Theorem 2.4 and Corollary 2.7]). We will show, in Section 5, that a helicoidal flat surface in $\mathbb{H}^{3}$ is characterized as a flat surface whose first and second fundamental forms are given by (3-1) and (3-2) where $\phi$ is linear, that is,

$$
\phi(u, v)=a u+b v+c,
$$

and $a, b$ and $c$ are real numbers such that $(a, b, c) \neq(0, \pm 1,0)$.

We will use the conformal representation for flat surfaces in $\mathbb{R}^{3}$ introduced in [Gálvez et al. 2000]. Let $\Sigma$ be a 2-manifold and $\psi: \Sigma \rightarrow \mathbb{H}^{3}$ a flat immersion. It follows from the Gauss equation that the second fundamental form $d \sigma^{2}$ is definite, and hence $\Sigma$ is orientable and inherits a canonical Riemann surface structure such that the second fundamental form $d \sigma^{2}$ is hermitian. This canonical Riemann surface structure provides a conformal representation for the immersion $\psi$ that allows one to recover any flat surface in $\mathbb{H}^{3}$ in terms of holomorphic data (see [Gálvez et al. 2000; Kokubu et al. 2004] for details). Throughout this paper, we will regard $\Sigma$ as a Riemann surface with the conformal structure determined by the second fundamental form $d \sigma^{2}$.

For any $p \in \Sigma$, there exist $g(p), g^{*}(p) \in \mathbb{C}_{\infty}$ distinct points in the ideal boundary such that the oriented normal geodesic at $\psi(p)$ is the geodesic in $\mathbb{H}^{3}$ starting from $g^{*}(p)$ towards $g(p)$. The maps $g, g^{*}: \Sigma \rightarrow \mathbb{C}_{\infty}$ are called the hyperbolic Gauss maps, and it is proved in [Gálvez et al. 2000] that, for flat surfaces, they are holomorphic when we regard $\mathbb{C}_{\infty}$ as the Riemann sphere.

Kokubu et al. [2004] extended the conformal representation given by Gálvez et al. [2000] for flat fronts, that is, flat immersions with some admissible singularities 
occurring where the first fundamental form degenerates. They showed how to recover flat fronts in terms of the hyperbolic Gauss maps and how these maps are well defined through the singularities. Reformulating the results in Theorem 2.11 and Proposition 2.5 of [Kokubu et al. 2004] to the upper half-space model, we have the following theorem (see [Corro et al. 2010]):

Theorem 1. Let $g$ and $g^{*}$ be nonconstant meromorphic functions on a Riemann surface $\Sigma$ such that $g(p) \neq g^{*}(p)$ for all $p \in \Sigma$. Assume that

(1) all the poles of the 1-form $\frac{d g}{g-g^{*}}$ are of order 1 , and

(2) $\operatorname{Re} \int_{\gamma} \frac{d g}{g-g^{*}}=0$ for each loop $\gamma$ on $\Sigma$.

Set

$$
\xi:=c \exp \int \frac{d g}{g-g^{*}}, \quad c \in \mathbb{C} \backslash\{0\} .
$$

Then the map $\psi=\left(\psi_{1}, \psi_{2}, \psi_{3}\right): \Sigma \rightarrow \mathbb{M}^{3}$ given by

$$
\psi_{1}+\mathrm{i} \psi_{2}=g-\frac{|\xi|^{4}\left(g-g^{*}\right)}{|\xi|^{4}+\left|g-g^{*}\right|^{2}}, \quad \psi_{3}=\frac{|\xi|^{2}\left|g-g^{*}\right|^{2}}{|\xi|^{4}+\left|g-g^{*}\right|^{2}}
$$

is a flat front. Moreover, if we consider the 1-forms

$$
\omega=-\frac{1}{\xi^{2}} g_{z} d z, \quad \theta=\frac{\xi^{2}}{\left(g-g^{*}\right)^{2}} g_{z}^{*} d z
$$

where $z$ is a complex parameter, then the first and second fundamental forms are represented as

$$
\begin{aligned}
\mathrm{I} & =(\omega+\bar{\theta})(\bar{\omega}+\theta), \\
\mathrm{II} & =|\theta|^{2}-|\omega|^{2} .
\end{aligned}
$$

The next proposition provides a necessary and sufficient condition on the functions $g$ and $g^{*}$ in order to diagonalize the first and second fundamental forms simultaneously:

Proposition 2. Let $\Sigma$ be a flat front in $\mathbb{H}^{3}$ given as in Theorem 1. A complex parameter for $\Sigma, \eta=u+\mathrm{i} v$, diagonalizes the first and second fundamental forms simultaneously as in (3-1) and (3-2) if and only if

$$
\frac{g_{\eta} g_{\eta}^{*}}{\left(g-g^{*}\right)^{2}}=-\frac{1}{4},
$$

where $(\cdot)_{\eta}$ is the derivative with respect to $\eta$. In this case, the harmonic function $\phi$ 
is given by

$$
e^{2 \phi}=\frac{\left|g_{\eta}^{*}\right||\xi|^{4}}{\left|g_{\eta}\right|\left|g-g^{*}\right|^{2}}=\frac{|\xi|^{4}}{4\left|g_{\eta}\right|^{2}} .
$$

Proof. It follows from (3-6) and (3-7) that

$$
I=|\omega|^{2}+|\theta|^{2}+2 \operatorname{Re}(\omega \theta)
$$

where

$$
\omega \theta=-\frac{g_{\eta} g_{\eta}^{*}(d \eta)^{2}}{\left(g-g^{*}\right)^{2}}
$$

since $g$ and $g^{*}$ are holomorphic functions on the parameter $\eta$.

By writing

$$
\frac{g_{\eta} g_{\eta}^{*}}{\left(g-g^{*}\right)^{2}}=A+\mathrm{i} B
$$

we have $\operatorname{Re}(\theta \omega)=-A\left(d u^{2}-d v^{2}\right)+2 B d u d v$. Then if $\eta$ diagonalizes the first and second fundamental forms as in (3-1) and (3-2), we must have $B=0$. Therefore $g_{\eta} g_{\eta}^{*} /\left(g-g^{*}\right)^{2}$ is real and holomorphic, which implies it must be a constant function.

If we write

$$
\frac{g_{\eta} g_{\eta}^{*}}{\left(g-g^{*}\right)^{2}}=c_{g}
$$

and use equations (3-6) and (3-11), we have the first fundamental form as in (3-1) if and only if

$$
\frac{\left|g_{\eta}\right|^{2}}{|\xi|^{4}}+\frac{|\xi|^{4}\left|g_{\eta}^{*}\right|^{2}}{\left|g-g^{*}\right|^{4}}-2 c_{g}=\cosh ^{2} \phi, \quad \frac{\left|g_{\eta}\right|^{2}}{|\xi|^{4}}+\frac{|\xi|^{4}\left|g_{\eta}^{*}\right|^{2}}{\left|g-g^{*}\right|^{4}}+2 c_{g}=\sinh ^{2} \phi
$$

Hence, $c_{g}=-\frac{1}{4}$ and (3-9) is proved.

Now we prove the expression (3-10). With this value for $c_{g}$, using equations (3-9) and (3-12), we rewrite the expressions above as

$$
\begin{aligned}
& \frac{\left|g_{\eta}\right|\left|g-g^{*}\right|^{2}}{4|\xi|^{4}\left|g_{\eta}^{*}\right|}+\frac{|\xi|^{4}\left|g_{\eta}^{*}\right|}{4\left|g_{\eta}\right|\left|g-g^{*}\right|^{2}}+\frac{1}{2}=\cosh ^{2} \phi, \\
& \frac{\left|g_{\eta}\right|\left|g-g^{*}\right|^{2}}{4|\xi|^{4}\left|g_{\eta}^{*}\right|}+\frac{|\xi|^{4}\left|g_{\eta}^{*}\right|}{4\left|g_{\eta}\right|\left|g-g^{*}\right|^{2}}-\frac{1}{2}=\sinh ^{2} \phi .
\end{aligned}
$$

Considering $\lambda=\frac{\left|g_{\eta}\right|\left|g-g^{*}\right|^{2}}{|\xi|^{4}\left|g_{\eta}^{*}\right|}$, we conclude that

$$
\left(\lambda+\frac{1}{\lambda}\right)=e^{2 \phi}+e^{-2 \phi} .
$$


If we now consider the second fundamental form, we have

$$
\begin{aligned}
\mathrm{II} & =|\theta|^{2}-|\omega|^{2} \\
& =\left(\frac{|\xi|^{4}\left|g_{\eta}^{*}\right|}{4\left|g-g^{*}\right|^{2}\left|g_{\eta}\right|}-\frac{\left|g-g^{*}\right|^{2}\left|g_{\eta}\right|}{4|\xi|^{4}\left|g_{\eta}^{*}\right|}\right)\left(d u^{2}+d v^{2}\right)=\frac{1}{4}\left(\frac{1}{\lambda}-\lambda\right)\left(d u^{2}+d v^{2}\right) .
\end{aligned}
$$

Therefore, it follows from (3-2) that we must have

$$
\left(\frac{1}{\lambda}-\lambda\right)=e^{2 \phi}-e^{-2 \phi}
$$

Combining (3-14) and (3-15), we conclude that

$$
e^{2 \phi}=\frac{1}{\lambda}=\frac{|\xi|^{4}\left|g_{\eta}^{*}\right|}{\left|g_{\eta}\right|\left|g-g^{*}\right|^{2}} .
$$

Corollary 3. Let $\Sigma$ be a flat front in $\mathbb{H}^{3}$. Two complex parameters for $\Sigma, z$ and $w$, diagonalize the first and second fundamental forms if and only if $w= \pm z+c$, where $c \in \mathbb{C}$ is a constant.

\section{Classifying examples}

In this section, we present an important class of examples of flat fronts whose hyperbolic Gauss maps are determined by a nonzero complex number. We call them classifying examples. We prove that if a flat front $\Sigma$ corresponds to a harmonic function $\phi$, then $\phi$ is linear if and only if $\Sigma$ is locally congruent to one of the classifying examples or to the peach front, which is a flat front presented in [Kokubu et al. 2005].

Theorem 4. For each $z_{0} \in \mathbb{C}^{*}=\mathbb{C} \backslash\{0\}$, consider $g: \mathbb{C} \rightarrow \mathbb{C}^{*}$, the holomorphic function given by

$$
g(z)=e^{\left(\varepsilon \sinh z_{0}\right) z}
$$

and $g^{*}=e^{2 z_{0}} g$. Then there exists a flat front $\psi_{z_{0}}: \mathbb{C} \rightarrow \mathbb{H}^{3}$ whose singular set is

$$
\mathscr{S}=\left\{z \in \mathbb{C} \mid \operatorname{Re}\left[\left(\varepsilon \cosh z_{0}\right) z\right]=0\right\}, \quad \varepsilon^{2}=-1 .
$$

Also, the first and second fundamental forms of the flat immersion $\psi_{z_{0}}: \mathbb{C} \backslash \mathscr{Y} \rightarrow \mathbb{M}^{3}$ can be written as in (3-1) and (3-2), where $\phi_{z_{0}}(z, \bar{z})$ is either a nonzero constant or

$$
\phi_{z_{0}}(u, v)=-\operatorname{Re}\left[\left(\varepsilon \cosh z_{0}\right) z\right]=a u+b v .
$$

Proof. From the definitions of $g$ and $g^{*}$ and since $z_{0} \neq 0$, it follows immediately that $g$ and $g^{*}$ are meromorphic nonconstant functions and $g \neq g^{*}$. Besides, we have

$$
\frac{d g}{g-g^{*}}=-\frac{\varepsilon d z}{2 e^{z_{0}}}
$$


which implies that the conditions (1) and (2) of Theorem 1 are satisfied. On the other hand,

$$
\frac{g_{z} g_{z}^{*}}{\left(g-g^{*}\right)^{2}}=\frac{e^{2 z_{0}}\left(g_{z}\right)^{2}}{\left(1-e^{2 z_{0}}\right)^{2} g^{2}}=\frac{\varepsilon^{2} e^{2 z_{0}} \sinh ^{2} z_{0}}{\left(1-e^{2 z_{0}}\right)^{2}}=-\frac{1}{4}
$$

By Theorem 1 and Proposition 2, there exists a flat front $\psi_{z_{0}}: \mathbb{C} \rightarrow \mathbb{H}^{3}$, given by $\psi_{z_{0}}=\left(\psi_{1}, \psi_{2}, \psi_{3}\right)$, with $\psi_{1}, \psi_{2}, \psi_{3}$ as in (3-4) and (3-5). Its first and second fundamental forms are as in (3-1) and (3-2). From the definition of $g$ and $g^{*}$ and Equations (3-4) and (3-10), it follows that $\phi_{z_{0}}(z, \bar{z})$ is either a nonzero constant if $\cosh z_{0}=0$, that is, $e^{z_{0}}= \pm \mathrm{i}$, or

$$
\phi(z, \bar{z})=-\operatorname{Re}\left(\varepsilon \cosh z_{0} z\right)=a u+b v, \quad a, b \in \mathbb{R} .
$$

In this last case, the singular set of $\psi_{z_{0}}$ is the straight line given by

$$
\mathscr{S}=\left\{z \in \mathbb{C} \mid \operatorname{Re}\left(\varepsilon \cosh z_{0} z\right)=0\right\} .
$$

Choosing all the possible values for $z_{0}$ in Theorem 4 , we obtain a family of examples that will provide the complete classification of the helicoidal flat surfaces. We will visualize the examples in the Poincaré ball model for $\mathbb{H}^{3}$.

(i) Rotational flat fronts. These flat fronts are obtained when $e^{2 z_{0}} \in \mathbb{R}$. The hyperbolic cylinder (Figure 1, left) is obtained when $e^{2 z_{0}}=-1$. When $e^{2 z_{0}}<0$ with $e^{2 z_{0}} \neq-1$ we have the hourglass (Figure 1, center), and for $e^{2 z_{0}}>0$ we have the snowman (Figure 1, right).

(ii) Conical flat fronts. This flat front (Figure 2) is obtained when $e^{2 z_{0}}= \pm \mathrm{i}$. In this case the invariance is only by the movement of translation.

(iii) Properly helicoidal flat fronts. The cases not mentioned above are invariant by the two movements, the rotational movement and the translation (Figure 3).

The class of examples obtained from Theorem 4 will be called classifying examples.
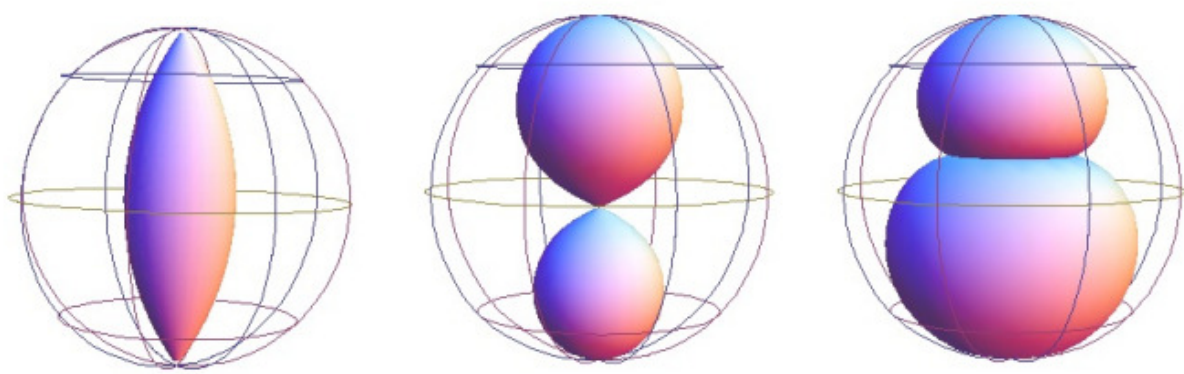

Figure 1. Rotational flat fronts: cylinder (left), hourglass (center), and snowman (right). 

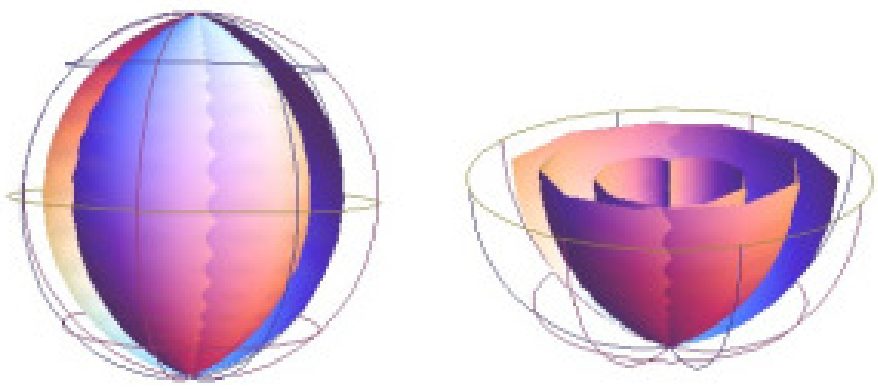

Figure 2. Conical flat fronts.
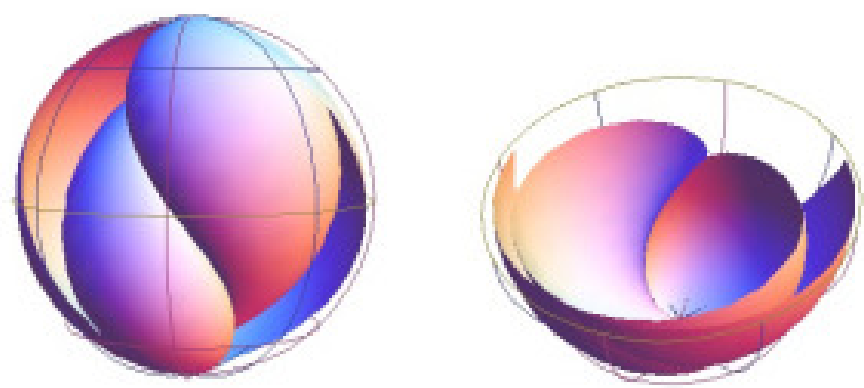

Figure 3. Properly helicoidal flat fronts.

Remark 5. The example given in [Kokubu et al. 2005], called the peach front (Figure 4), is a case where the hyperbolic Gauss maps satisfy $g^{*}=g-1$, and it can be parametrized using Theorem 1 as

$$
\left(\psi_{1}, \psi_{2}, \psi_{3}\right)=\left( \pm \frac{v}{2}-\frac{e^{ \pm 2 v}}{e^{ \pm 2 v}+1}, \mp \frac{u}{2}, \frac{e^{ \pm v}}{e^{ \pm 2 v}+1}\right)
$$

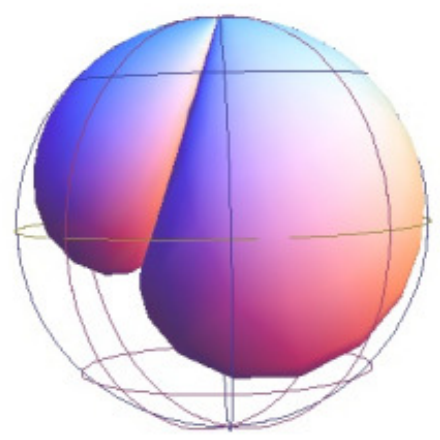

Figure 4. The peach front. 
where the first and second fundamental forms are given as (3-1) and (3-2) with $\phi(u, v)= \pm v$. Observe that this value of $\phi$ can be viewed as

$$
\phi(z, \bar{z})=-\operatorname{Re}\left(\varepsilon \cosh z_{0} z\right)
$$

with $z_{0}=0$.

Theorem 6. Let $\Sigma \subset \mathbb{M}^{3}$ be a flat front with a complex parameter $z=u+\mathrm{i} v$ that diagonalizes the first and second fundamental forms simultaneously as in (3-1) and (3-2). Then $\phi(u, v)=a u+b v+c$ if and only if the corresponding flat front is locally congruent to one of the classifying examples or to a peach front.

Proof. Since $\phi$ and $z=u+\mathrm{i} v$ determine the first and second fundamental forms, it is clear that any flat immersion such that $\phi(z, \bar{z})=-\operatorname{Re}\left(\varepsilon \cosh z_{0} z\right)$, for some $z_{0} \in \mathbb{C}^{*}$, must be locally congruent to one of the classifying examples.

On the other hand, as we observed in Remark 5, the peach front has a parametrization that diagonalizes the first and second fundamental forms with $\phi(u, v)$ as above, with $z_{0}=0$.

\section{Characterization}

In this section, we prove that a flat front in $\mathbb{H}^{3}$ is helicoidal if and only if it is locally congruent to one of the classifying examples presented in the previous section. Moreover, we obtain a complete classification of the helicoidal flat fronts in terms of their hyperbolic Gauss maps, as well as by means of linear harmonic functions. As a consequence of Theorem 6, we prove that any flat surface in $\mathbb{H}^{3}$ that corresponds to a linear harmonic function is locally congruent either to a helicoidal flat surface or to a peach front.

Theorem 7. A flat front in $\mathbb{H}^{3}$ is helicoidal if and only if it is locally congruent to one of the classifying examples.

We split our proof into two lemmas. The first lemma will establish that every classifying example has the geometric property that it is invariant by a helicoidal group of isometries, that is, it is a helicoidal flat front. In the second, we will show that, given any helicoidal flat front, there exists a rigid motion of $\mathbb{H}^{3}$ such that its hyperbolic Gauss maps satisfy $g^{*}=e^{2 z_{0}} g$, where $z_{0}$ is a nonzero complex number. Once we establish these two lemmas, the proof of Theorem 7 will follow as a consequence of Proposition 2 and Theorem 6.

Lemma 8. Every classifying example is a helicoidal flat front.

Proof. The classifying examples were obtained by using the method of producing flat fronts given by Theorem 4. Given such a flat front, its hyperbolic Gauss maps $g$ and $g *$ satisfy $g *=e^{2 z_{0}} g$ and $g=e^{\left(\varepsilon \sinh z_{0}\right) z}$, where $z_{0}$ is a nonzero complex 
number, that is, $1-e^{z_{0}} \neq 0$. We want to obtain the immersion in $\mathbb{M}^{3}$ of the flat front, associated to $g$ and $g *$, by using Theorem 1 . Since $g *=e^{2 z_{0}} g$, we have

$$
g-g^{*}=\left(1-e^{2 z_{0}}\right) g .
$$

Setting $g=R e^{\mathrm{i} v}$, it follows from (3-4) that

$$
\xi=c \exp \left(\frac{\log R+\mathrm{i} v}{1-e^{2 z_{0}}}\right) .
$$

From now on, we adopt the notation

$$
\frac{1}{1-e^{2 z_{0}}}=x_{0}+\mathrm{i} y_{0} .
$$

Then we have

$$
|\xi|^{2}=|c|^{2} e^{2\left(x_{0} \log R-y_{0} v\right)} .
$$

We can now obtain the flat front given by (3-5). Using (5-1) and (5-2), we have

$$
\begin{aligned}
\psi_{1}+\mathrm{i} \psi_{2} & =\left(1-\frac{|c|^{4} e^{4\left(x_{0} \log R-y_{0} v\right)}\left(x_{0}-\mathrm{i} y_{0}\right)}{|c|^{4} e^{4\left(x_{0} \log R-y_{0} v\right)}\left(x_{0}^{2}+y_{0}^{2}\right)+e^{(2 \log R)}}\right) R e^{\mathrm{i} v}, \\
\psi_{3} & =\frac{|c|^{2} e^{2\left(x_{0} \log R-y_{0} v+\log R\right)}}{\left(x_{0}^{2}+y_{0}^{2}\right)|c|^{4} e^{4\left(x_{0} \log R-y_{0} v\right)}+e^{2 \log R}} .
\end{aligned}
$$

Simplifying (5-4), we have

$$
\begin{aligned}
\psi_{3} & =\frac{|c|^{2} R e^{\left(2 x_{0} \log R-2 y_{0} v+\log R\right)}}{\left(x_{0}^{2}+y_{0}^{2}\right)|c|^{4} e^{4\left(x_{0} \log R-y_{0} v\right)}+e^{2 \log R}} \\
& =\frac{|c|^{2} R}{\left(x_{0}^{2}+y_{0}^{2}\right)|c|^{4} e^{\left(2 x_{0} \log R-2 y_{0} v-\log R\right)}+e^{\left(-2 x_{0} \log R+2 y_{0} v+\log R\right)}} \\
& =\frac{|c|^{2} R}{\left(x_{0}^{2}+y_{0}^{2}\right)|c|^{4} e^{x}+e^{-x}},
\end{aligned}
$$

where $x=\left(2 x_{0}-1\right) \log R-2 y_{0} v$. Using this fact, we rewrite (5-3):

(5-6) $\quad \psi_{1}+\mathrm{i} \psi_{2}$

$$
\begin{aligned}
& =\left(1-\frac{|c|^{4} e^{\left(4 x_{0} \log R-4 y_{0} v\right)}\left(x_{0}-\mathrm{i} y_{0}\right)}{\left(x_{0}^{2}+y_{0}^{2}\right)|c|^{4} e^{\left(4 x_{0} \log R-4 y_{0} v\right)}+e^{(2 \log R)}}\right) R e^{\mathrm{i} v} \\
& =\left(1-\frac{|c|^{4} e^{\left(2 x_{0} \log R-2 y_{0} v-\log R\right)}\left(x_{0}-\mathrm{i} y_{0}\right)}{\left(x_{0}^{2}+y_{0}^{2}\right)|c|^{4} e^{\left(2 x_{0} \log R-2 y_{0} v-\log R\right)}+e^{\left(-2 x_{0} \log R+2 y_{0} v+\log R\right)}}\right) R e^{\mathrm{i} v} \\
& =\left(1-\frac{|c|^{4}\left(x_{0}-\mathrm{i} y_{0}\right) e^{x}}{\left(x_{0}^{2}+y_{0}^{2}\right)|c|^{4} e^{x}+e^{-x}}\right) R e^{\mathrm{i} v} .
\end{aligned}
$$


Now we want to prove that the immersed surface is invariant by the helicoidal group of isometries of $\mathbb{M}^{3}$. First, let us consider the case when $y_{0}=0$. We can see from (5-5) and (5-6) that this case corresponds to the rotational surfaces. On the other hand, when $y_{0} \neq 0$, we can write

$$
v=f(R)-\frac{x}{2 y_{0}},
$$

where $f(R)=\frac{2 x_{0}-1}{2 y_{0}} \log R$. With this notation, we obtain

$$
\begin{aligned}
& \psi_{1}=R\left(c_{1}(x) \cos f(R)-c_{2}(x) \sin f(R)\right), \\
& \psi_{2}=R\left(c_{1}(x) \sin f(R)+c_{2}(x) \cos f(R)\right),
\end{aligned}
$$

where $c_{1}$ and $c_{2}$ are real functions given by

$$
\begin{aligned}
& c_{1}(x)=\frac{\left(\left(x_{0}^{2}+y_{0}^{2}-x_{0}\right)|c|^{4} e^{x}+e^{-x}\right) \cos \frac{x}{2 y_{0}}+y_{0}|c|^{4} e^{x} \sin \frac{x}{2 y_{0}}}{\left(x_{0}^{2}+y_{0}^{2}\right)|c|^{4} e^{x}+e^{-x}}, \\
& c_{2}(x)=-\frac{\left(\left(x_{0}^{2}+y_{0}^{2}-x_{0}\right)|c|^{4} e^{x}+e^{-x}\right) \sin \frac{x}{2 y_{0}}+y_{0}|c|^{4} e^{x} \cos \frac{x}{2 y_{0}}}{\left(x_{0}^{2}+y_{0}^{2}\right)|c|^{4} e^{x}+e^{-x}} .
\end{aligned}
$$

Using the notation $\psi_{3}=R c_{3}(x)$, we then have the following expression for the front:

$$
\left(\psi_{1}, \psi_{2}, \psi_{3}\right)(R, x)=R\left(\begin{array}{ccc}
\cos f(R) & -\sin f(R) & 0 \\
\sin f(R) & \cos f(R) & 0 \\
0 & 0 & 1
\end{array}\right)\left(\begin{array}{l}
c_{1}(x) \\
c_{2}(x) \\
c_{3}(x)
\end{array}\right)
$$

When $x_{0}=\frac{1}{2}$, we have $f(R)=0$, and consequently there is no rotational movement. On the other hand, if $x_{0} \neq \frac{1}{2}$, we consider $f(R)=y$ and write

$$
\left(\psi_{1}, \psi_{2}, \psi_{3}\right)(y, x)=\exp \left(\frac{2 y_{0}}{2 x_{0}-1} y\right)\left(\begin{array}{ccc}
\cos y & -\sin y & 0 \\
\sin y & \cos y & 0 \\
0 & 0 & 1
\end{array}\right)\left(\begin{array}{l}
c_{1}(x) \\
c_{2}(x) \\
c_{3}(x)
\end{array}\right) .
$$

This concludes the proof of the lemma.

The second lemma will show that any helicoidal flat surface in $\mathbb{H}^{3}$ is congruent to a surface whose hyperbolic Gauss maps satisfy $g^{*}=c g$, where $c$ is a complex number.

In order to do so, we will consider an approach closer to the one given in [Ripoll 1989]. We consider hyperbolic space $\mathbb{H}^{3}$ as a submanifold of the Lorentzian 4-space $\mathbb{L}^{4}$, endowed with coordinates $\left(x_{0}, x_{1}, x_{2}, x_{3}\right)$ and the inner product $\langle$,$\rangle given by$

$$
\langle,\rangle=-d x_{0}^{2}+d x_{1}^{2}+d x_{2}^{2}+d x_{3}^{2} .
$$


The hyperbolic 3-space $\mathbb{M}^{3}$ will be the Riemannian 3-submanifold with sectional curvature -1 given by the set

$$
\mathbb{M}^{3}=\left\{\left(x_{0}, x_{1}, x_{2}, x_{3}\right) \in \mathbb{L}^{4} \mid-x_{0}^{2}+x_{1}^{2}+x_{2}^{2}+x_{3}^{2}=-1, x_{0}>0\right\},
$$

with the metric induced by $\mathbb{L}^{4}$.

We can see that the map

$$
\left(x_{0}, x_{1}, x_{2}, x_{3}\right) \rightarrow \frac{1}{x_{0}+x_{3}}\left(x_{1}, x_{2}, 1\right)
$$

is an isometry between this model and the half-space model. Its inverse is given by

$$
\left(y_{1}, y_{2}, y_{3}\right) \rightarrow \frac{1}{2 y_{3}}\left(1+\sum_{i=1}^{3} y_{i}^{2}, 2 y_{1}, 2 y_{2}, 1-\sum_{i=1}^{3} y_{i}^{2}\right) \text {. }
$$

With these maps in mind we consider the helicoidal flat surfaces in $\mathbb{M}^{3} \subset \mathbb{L}^{4}$. Let $O_{1}(4)$ be the orthogonal group in $\mathbb{L}^{4}$ given by all linear transformations that preserve $\langle$,$\rangle . Now consider m_{t} \in O_{1}(4)$ given by the matrix

$$
m_{t}=\left(\begin{array}{cccc}
\cosh \beta t & 0 & 0 & \sinh \beta t \\
0 & \cos \alpha t & -\sin \alpha t & 0 \\
0 & \sin \alpha t & \cos \alpha t & 0 \\
\sinh \beta t & 0 & 0 & \cosh \beta t
\end{array}\right) .
$$

Observe that $m_{t}$ is a one-parameter subgroup of isometries of $\mathbb{M}^{3}$ given by a translation

$$
\left(\begin{array}{cccc}
\cosh \beta t & 0 & 0 & \sinh \beta t \\
0 & 1 & 0 & 0 \\
0 & 0 & 1 & 0 \\
\sinh \beta t & 0 & 0 & \cosh \beta t
\end{array}\right)
$$

along the geodesic $\gamma:-x_{0}^{2}+x_{3}^{2}=-1$, composed with the rotation

$$
\left(\begin{array}{cccc}
1 & 0 & 0 & 0 \\
0 & \cos \alpha t & -\sin \alpha t & 0 \\
0 & \sin \alpha t & \cos \alpha t & 0 \\
0 & 0 & 0 & 1
\end{array}\right) .
$$

Also, observe that the geodesic $\gamma$ we are considering is the image of the $y_{3}$-axis by the map (5-8). One verifies that any orbit of $m_{t}$ intersects the totally geodesic submanifold $P^{2}=\left\{x_{3}=0\right\}$ just once. Thus, up to congruences, any surface invariant under $m_{t}$ is generated by a curve in $P^{2}$.

In order to obtain the hyperbolic Gauss maps, we now consider $\mathbb{H}^{3}$ contained in the Lorentzian 4-space $\mathbb{L}^{4}$. We use the theory developed in [Gálvez et al. 2000], where there is a description of these maps using an identification between $\mathbb{L}^{4}$ and 


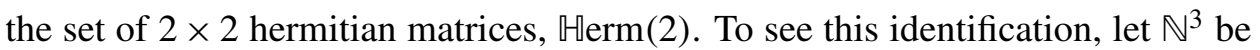
the half part of the light cone such that $x_{0}>0$,

$$
\mathbb{N}^{3}=\left\{\left(x_{0}, x_{1}, x_{2}, x_{3}\right) \in \mathbb{L}^{4} \mid-x_{0}^{2}+x_{1}^{2}+x_{2}^{2}+x_{3}^{2}=0, x_{0}>0\right\} .
$$

If we associate to each $v \in \mathbb{N}^{3}$ the half-line [ $v$ ], we obtain a partition of $\mathbb{N}^{3}$, and the ideal boundary $\mathbb{S}_{\infty}^{2}$ of $\mathbb{\boxplus}^{3}$ can be viewed as the quotient of $\mathbb{N}^{3}$ under the associated equivalence relation. Thus, the induced metric is well defined up to a scalar multiple, where $\mathbb{S}_{\infty}^{2}$ receives a natural conformal structure as the quotient $\mathbb{N}^{3} / \mathbb{R}^{+}$. In this approach, as we can see in [Gálvez et al. 2000], the hyperbolic Gauss maps of an immersion $\psi: S \rightarrow \mathbb{M}^{3}$ with unit normal vector field $N$ are given by

$$
g=[\psi+N] \text { and } g^{*}=[\psi-N] .
$$

We use the identification between $\mathbb{L}^{4}$ and the set of $2 \times 2$ hermitian matrices Herm(2), where the point $\left(x_{0}, x_{1}, x_{2}, x_{3}\right)$ is identified with the matrix

$$
\left(\begin{array}{cc}
x_{0}+x_{3} & x_{1}+\mathrm{i} x_{2} \\
x_{1}-\mathrm{i} x_{2} & x_{0}-x_{3}
\end{array}\right)
$$

Once we have the coordinates of $\psi+N$ and $\psi-N$ in $\mathbb{L}^{4}$, we find their corresponding matrices and write them as

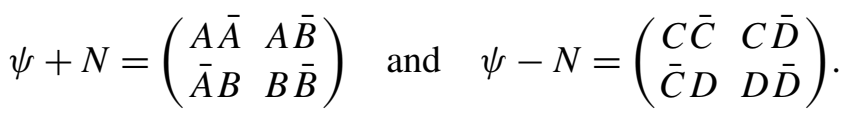

Therefore, we have the hyperbolic Gauss maps given by

$$
g=\frac{A}{B} \quad \text { and } \quad g^{*}=\frac{C}{D}
$$

(see [Gálvez et al. 2000] for more details).

With this approach, we are able to establish and prove the second lemma:

Lemma 9. Let $\psi: \sigma \rightarrow \mathbb{H}^{3}$ be an immersion of a helicoidal flat surface in $\mathbb{H}^{3}$. Then there is a rigid motion of $\mathbb{U}^{3}$ such that its hyperbolic Gauss maps $g$ and $g^{*}$ satisfy $g^{*}=e^{2 z_{0}} g$, where $z_{0} \neq 0$ is a complex number.

Proof. We start with a helicoidal surface immersed in $\mathbb{M}^{3}$. Then considering the half-space model for $\mathbb{T}^{3}$, there exists a rigid motion of $\mathbb{\boxplus}^{3}$ that takes the axis of the helicoidal surface into the $y_{3}$-axis. Then by considering the isometry (5-8), between the half-space model and $\mathbb{H}^{3} \subset \mathbb{L}^{4}$, up to a rigid motion of $\mathbb{M}^{3}$, we may consider the immersion $\psi$ of the helicoidal surface as

$$
\psi(t, s)=m_{t}(\gamma(s)),
$$

where $\gamma$ is a curve in $P^{2}$ parametrized by arc length. 
In order to describe the hyperbolic Gauss maps $g$ and $g^{*}$, we need to obtain the maps $\psi+N$ and $\psi-N$. A normal unit vector field is given by

$$
N=\frac{\otimes\left(\psi, \psi_{t}, \psi_{s}\right)}{\left|\bigotimes\left(\psi, \psi_{t}, \psi_{s}\right)\right|},
$$

where $\otimes\left(\psi, \psi_{t}, \psi_{s}\right)$ is the Lorentzian vector product between $\psi, \psi_{t}, \psi_{s}$. If we write $\gamma(s)=\left(x_{0}(s), x_{1}(s), x_{2}(s), 0\right)$, we have $\psi_{t}(t, s)=m_{t}(v(s))$, with the vector $v$ given by $v(s)=\left(0,-\alpha x_{2}(s), \alpha x_{1}(s), \beta x_{0}(s)\right)$. This fact and the orthogonality of $m_{t}$ enable us to conclude that

$$
N(t, s)=m_{t}(\eta(s)),
$$

where $\eta(s)=\frac{\bigotimes\left(\gamma(s), v(s), \gamma^{\prime}(s)\right)}{\left|\otimes\left(\gamma(s), v(s), \gamma^{\prime}(s)\right)\right|}$. Therefore

$$
\psi+N=m_{t}(\gamma+\eta), \quad \psi-N=m_{t}(\gamma-\eta) .
$$

From (5-11)-(5-12), we have

$$
g(s, t)=g_{0}(s) e^{(\beta+\mathrm{i} \alpha) t} \quad \text { and } \quad g^{*}(s, t)=g_{0}^{*}(s) e^{(\beta+\mathrm{i} \alpha) t} .
$$

Now we see that $g / g^{*}$ is a function only of the variable $s$, and as was proved in [Gálvez et al. 2000], $g$ and $g^{*}$ are holomorphic when the surface is flat. Therefore, $g / g^{*}$ is a holomorphic function that depends only on one variable, which implies

$$
g=\omega_{0} g^{*},
$$

where $\omega_{0} \in \mathbb{C}$ is a constant. It follows from (5-11)-(5-12) and the fact that $\psi$ and $N$ are orthogonal that $\omega_{0} \neq 1$. Therefore, there exists $z_{0} \in \mathbb{C}^{*}$ such that $g=e^{2 z_{0}} g^{*}$.

Proof of Theorem 7. One direction of the proof is given by Lemma 8, that is, every classifying example is a helicoidal flat front. Conversely, given any helicoidal flat surface in $\mathbb{H}^{3}$, it follows from Lemma 9 that it is congruent to a surface whose hyperbolic Gauss maps must satisfy $g=e^{2 z_{0}} g^{*}$, where $z_{0} \neq 0$. Moreover, using Proposition 2, we can choose a complex parameter $\eta=u+\mathrm{i} v$ such that (3-9) holds. Therefore, locally $g$ is given by

$$
g=e^{\varepsilon\left(\sinh z_{0}\right) \eta} .
$$

Then it follows from (3-10) that $\phi$ must be linear. Theorem 6 implies that this helicoidal flat front is locally congruent to one of the classifying examples.

As a consequence of Theorem 7, Proposition 2, Theorem 4 and the definition of the classifying examples, we have a complete classification of the helicoidal flat fronts in terms of their hyperbolic Gauss maps, determined by a nonzero complex number. 
Theorem 10. A flat front in $\mathbb{M}^{3}$ is helicoidal if and only if up to a rigid motion of $\mathbb{M}^{3}$, there exists a complex parameter $\eta$ such that its hyperbolic Gauss maps $g$ and $g^{*}$ are meromorphic functions given by

$$
g=e^{\varepsilon\left(\sinh z_{0}\right) \eta} \text { and } g^{*}=e^{2 z_{0}} g,
$$

where $z_{0}$ is a nonzero complex number and $\varepsilon^{2}=1$.

As a consequence of Theorems 4, 7 and 10, we get the following two results formulated in term of harmonic functions.

Theorem 11. A flat front in $\mathbb{H}^{3}$ is helicoidal if and only if there exists a local parametrization by lines of curvature in a neighborhood of a nonsingular point, such that the first and second fundamental forms are given by (3-1) and (3-2), where

$$
\phi=a u+b v+c \quad \text { and } \quad(a, b, c) \neq(0, \pm 1,0) .
$$

Theorem 12. Let $\Sigma$ be a flat front in $\mathbb{H}^{3}$ with a local parametrization in a neighborhood of a nonsingular and nonumbilic point, such that the first and second fundamental forms are diagonal and given by (3-1) and (3-2), where $\phi$ is a (euclidean) harmonic function. Then $\phi$ is linear, that is, $\phi=a u+b v+c$ if and only if $\Sigma$ is locally congruent either to a helicoidal flat front or to a peach front.

\section{References}

[do Carmo and Dajczer 1983] M. do Carmo and M. Dajczer, "Rotation hypersurfaces in spaces of constant curvature", Trans. Amer. Math. Soc. 277:2 (1983), 685-709. MR 85b:53055 Zbl 0518.53059

[Corro et al. 2010] A. V. Corro, A. Martínez, and F. Milán, "Complete flat surfaces with two isolated singularities in hyperbolic 3-space", J. Math. Anal. Appl. 366:2 (2010), 582-592. MR 2011b:53028 Zbl 1190.53055

[Gálvez et al. 2000] J. A. Gálvez, A. Martínez, and F. Milán, "Flat surfaces in the hyperbolic 3-space", Math. Ann. 316:3 (2000), 419-435. MR 2002b:53013 Zbl 1003.53047

[Kokubu et al. 2004] M. Kokubu, M. Umehara, and K. Yamada, "Flat fronts in hyperbolic 3-space", Pacific J. Math. 216:1 (2004), 149-175. MR 2005f:53021 Zbl 1078.53009

[Kokubu et al. 2005] M. Kokubu, W. Rossman, K. Saji, M. Umehara, and K. Yamada, "Singularities of flat fronts in hyperbolic space", Pacific J. Math. 221:2 (2005), 303-351. MR 2006k:53102 Zbl 1110.53044

[Ripoll 1989] J. B. Ripoll, "Helicoidal minimal surfaces in hyperbolic space", Nagoya Math. J. 114 (1989), 65-75. MR 91a:53015 Zbl 0699.53067

[Spivak 1979] M. Spivak, A comprehensive introduction to differential geometry, vol. 2, 2nd ed., Publish or Perish, Berkeley, CA, 1979. MR 82g:53003b Zbl 0439.53002

[Tenenblat 1998] K. Tenenblat, Transformations of manifolds and applications to differential equations, Pitman Monographs and Surveys in Pure and Applied Mathematics 93, Longman, Harlow, 1998. MR 2002b:37111 Zbl 0912.58045

Received February 29, 2012. Revised April 20, 2012. 
ANTONIO MARTÍNEZ

DEPARTAMENTO DE GEOMETRÍA Y TOPOLOGÍA

UNIVERSIDAD DE GRANADA

E-18071 GRANADA

SPAIN

amartine@ugr.es

Jõ̃o PaUlo dos SANTOS

DEPARTAMENTO DE MATEMÁTICA

UNIVERSIDADE DE BRASÍLIA

70910-900 BRASÍLIA

BRAZIL

joaopsantos@unb.br

Keti TENENBlat

Departamento de Matemática

UNIVERSIDADE DE BRASÍLIA

70910-900 BRASÍLIA

BRAZIL

k.tenenblat@mat.unb.br 


\title{
PACIFIC JOURNAL OF MATHEMATICS
}

\author{
msp.org/pjm
}

Founded in 1951 by E. F. Beckenbach (1906-1982) and F. Wolf (1904-1989)

\section{EDITORS}

V. S. Varadarajan (Managing Editor)

Department of Mathematics

University of California

Los Angeles, CA 90095-1555

pacific@math.ucla.edu

Paul Balmer

Department of Mathematics

University of California

Los Angeles, CA 90095-1555

balmer@math.ucla.edu

Daryl Cooper

Department of Mathematics

University of California

Santa Barbara, CA 93106-3080 cooper@math.ucsb.edu

Jiang-Hua $\mathrm{Lu}$

Department of Mathematics

The University of Hong Kong

Pokfulam Rd., Hong Kong jhlu@maths.hku.hk
Don Blasius

Department of Mathematics University of California

Los Angeles, CA 90095-1555

blasius@math.ucla.edu

Robert Finn

Department of Mathematics Stanford University

Stanford, CA 94305-2125

finn@math.stanford.edu

Sorin Popa

Department of Mathematics

University of California

Los Angeles, CA 90095-1555

popa@math.ucla.edu

Paul Yang

Department of Mathematics

Princeton University

Princeton NJ 08544-1000

yang@math.princeton.edu

\section{PRODUCTION}

Silvio Levy, Scientific Editor, production@msp.org

\section{SUPPORTING INSTITUTIONS}

ACADEMIA SINICA, TAIPEI

CALIFORNIA INST. OF TECHNOLOGY

INST. DE MATEMÁTICA PURA E APLICADA

KEIO UNIVERSITY

MATH. SCIENCES RESEARCH INSTITUTE

NEW MEXICO STATE UNIV.

OREGON STATE UNIV.

\author{
STANFORD UNIVERSITY \\ UNIV. OF BRITISH COLUMBIA \\ UNIV. OF CALIFORNIA, BERKELEY \\ UNIV. OF CALIFORNIA, DAVIS \\ UNIV. OF CALIFORNIA, LOS ANGELES \\ UNIV. OF CALIFORNIA, RIVERSIDE \\ UNIV. OF CALIFORNIA, SAN DIEGO \\ UNIV. OF CALIF., SANTA BARBARA
}

\author{
Vyjayanthi Chari \\ Department of Mathematics \\ University of California \\ Riverside, CA 92521-0135 \\ chari@math.ucr.edu \\ Kefeng Liu \\ Department of Mathematics \\ University of California \\ Los Angeles, CA 90095-1555 \\ liu@math.ucla.edu \\ Jie Qing \\ Department of Mathematics \\ University of California \\ Santa Cruz, CA 95064 \\ qing@cats.ucsc.edu
}

These supporting institutions contribute to the cost of publication of this Journal, but they are not owners or publishers and have no responsibility for its contents or policies.

See inside back cover or msp.org/pjm for submission instructions.

The subscription price for 2013 is US \$400/year for the electronic version, and \$485/year for print and electronic.

Subscriptions, requests for back issues and changes of subscribers address should be sent to Pacific Journal of Mathematics, P.O. Box 4163, Berkeley, CA 94704-0163, U.S.A. The Pacific Journal of Mathematics is indexed by Mathematical Reviews, Zentralblatt MATH, PASCAL CNRS Index, Referativnyi Zhurnal, Current Mathematical Publications and the Science Citation Index.

The Pacific Journal of Mathematics (ISSN 0030-8730) at the University of California, c/o Department of Mathematics, 798 Evans Hall \#3840, Berkeley, CA 94720-3840, is published monthly except July and August. Periodical rate postage paid at Berkeley, CA 94704, and additional mailing offices. POSTMASTER: send address changes to Pacific Journal of Mathematics, P.O. Box 4163, Berkeley, CA 94704-0163.

PJM peer review and production are managed by EditFLOW ${ }^{\circledR}$ from Mathematical Sciences Publishers.

PUBLISHED BY

mathematical sciences publishers

nonprofit scientific publishing

http://msp.org/

(C) 2013 Mathematical Sciences Publishers 


\section{PACIFIC JOURNAL OF MATHEMATICS}

Volume $264 \quad$ No. $1 \quad$ July 2013

On the center of fusion categories

ALAIN BRUGUIÈRES and ALEXIS VIRELIZIER

Connected quandles associated with pointed abelian groups

W. EdWin Clark, MoHamed ElHamdadi, Xiang-DONG HoU,

MASAHICO SAITO and TIMOTHY YEATMAN

Entropy and lowest eigenvalue on evolving manifolds

HongXin GuO, ROBERT PHILIPOWSKI and ANTON THALMAIER

Poles of certain residual Eisenstein series of classical groups

DIHUA JIANG, BAIYING LIU and LEI ZHANG

Harmonic maps on domains with piecewise Lipschitz continuous metrics 125

HAIGANG LI and CHANGYOU WANG

$q$-hypergeometric double sums as mock theta functions

JEREMY LOVEJOY and ROBERT OSBURN

Monic representations and Gorenstein-projective modules

XIU-HuA LUO and PU ZHANG

Helicoidal flat surfaces in hyperbolic 3-space

Antonio Martínez, JoÃo Paulo dos Santos and Keti

TENENBLAT

On a Galois connection between the subfield lattice and the multiplicative subgroup lattice

JOHN K. MCVEY

Some characterizations of Campanato spaces via commutators on Morrey 221 spaces

Shaoguang Shi and Shanzhen Lu

The Siegel-Weil formula for unitary groups 